Kalpa Publications in Engineering
Volume 4, 2022, Pages 122-130
Proceedings of International Sym-
posium on Applied Science 2021

\title{
Acne Scan - Application In Assessment Of Acne And Supports Treatment Road
}

\author{
Nguyen Xuan Nguyen Pham ${ }^{1,2^{*}}$, Thi Tham Tran ${ }^{1,2^{*}}$, Minh Thang Do ${ }^{1,2^{*}}$, \\ Ngoc Bao Duy Tran ${ }^{1,2^{*}}$ \\ ${ }^{1}$ Faculty of Computer Science and Engineering, Ho Chi Minh CityUniversity of Technology \\ (HCMUT), 268 Ly Thuong Kiet Street, ward 14, District 10, Ho Chi Minh City, Vietnam \\ ${ }^{2}$ Vietnam National University Ho Chi Minh City, Linh Trung Ward, Thu Duc District, Ho Chi \\ Minh City, Vietnam \\ nguyen.phamenttk17@hcmut.edu.vn, tham.trantranthambk@hcmut.eu.vn, \\ thang.do.at@hcmut.edu.vn, duytnb@hcmut.edu.vn
}

\begin{abstract}
As society develops, many aspects of life are concerned by people, including facial skincare, avoiding acne-related diseases. In this work, we will propose a complete solution for treating acne at home, including 4 processors. First, the anomaly detector uses image processing techniques by Multi-Threshold and Color Segmentation, depending on each color channel corresponding to each type of acne. The sensitivity of the detector is $89.4 \%$. Second, the set of anomalies classifiers into 6 main categories, including 4 major acne types and 2 non-acne types. By applying the convolutional neural model, the accuracy, sensitivity, and F1 are $84.17 \%, 81.5 \%$, and $82 \%$, respectively. Third, the acne status assessment kit is based on the mGAGS method to classify the condition of a face as mild, moderate, severe, or very severe with an accuracy of $81.25 \%$. Finally, the product recommender, which generalizes from the results of the previous processors with an accuracy of $70-90 \%$. This is the premise that helps doctors as well as general users to evaluate the level of acne on a face effectively and save time.
\end{abstract}

Keywords: Acne detection, Acne classification, Acne status assessment, Suggestion system.

\section{Introduction}

Acne is a chronic skin disease, very common and common in adolescents, with little impact on health but greatly affects aesthetics, psychology and quality of life. According to statistics of the National Hospital of Dermatology in 3 years (2007 - 2009), the number of acne patients coming to the

\footnotetext{
${ }^{*}$ Corresponding author. All authors contributed equally to this work.
} 
clinic accounted for $13.6 \%$ of the total number of skin diseases (Sáu, 2010). In particular, acne presents diversely with many different lesions: acne micronuclei, white-headed nucleus, black-headed nucleus, papules, etc.

Research by Amini and colleagues (Mohammad Amini, 22 February 2018) used the a* channel in the CIE L*a*b* color space to detect and classify inflammatory acne (red inflammatory papules and pustules). can detect $93 \%$ of acne lesions (in the ROI area) with different lighting conditions and skin color differences as well. Most of Amini's studies and previous studies detected skin lesions by processing 1 channel of the color space, but so only 1 type of acne lesion was detected. At the same time, the articles have not extracted 4 types of acne (whiteheads, blackheads, papules, pustules) from images with different lighting conditions and skin colors.

In recent years, the problem of acne classification has received more attention. In 2018, Xiaolei Shen, Jiachi Zhang, Chenjun Yan \& Hong Zhou (Xiaolei Shen, 11 April 2018) classified acne by the model proposed by the authors and used the Transfer Learning method by pretrain model VGG16. The accuracy is quite high from $81 \%-95 \%$. However, the method still has certain limitations. In 2019, Jung Cheeoh and colleagures (Cheeoh, 30 April 2019) from Korea also proposed a model to classify acne skin with $96 \%$ accuracy with input images of $224 \times 224 \times 3$. However, most of the prior studies included a standardized dataset that was reviewed by reputable dermatologists.

Acne severity is an indicator that dermatologists use to prescribe medications to treat acne lesions. Although there is no universally accepted classification system, several studies have attempted to find an appropriate criterion for the classification of acne lesions on the basis of the two proposed methods. published as "Global Acne Classification System" (R. Ramli, Feb. 2012) and "Hayashi Acne Counting System" (Nobukazu Hayashi, 2008).

In 2020, Gyeongeun Lee and associates proposed a content-based skin care product recommendation system (Gyeongeun Lee, 2020). This proposal focuses on designing a skin care product recommendation system based on user skin type and product ingredient composition. Contentbased filtering is used to identify product chemical compositions and find products with similar ingredients. The filtering method gives $75 \%$ higher average accuracy, so it's an efficient way to recommend the right product to the user, as long as $50 \%$ is neutral. But the results came from only a small sample, and so further investigation is needed to get a more reliable accuracy.

In this study, we will propose a 4-step method with each step being a significant improvement from previous works.

\section{Proposed Method}

\subsection{Abnormal Points Detection on Human Face Image}

Face recognition is the first step of the method. An image is recognized as an image that must meet the following criteria:

1. The photo is a photo of a person's face taken from the front camera,

2. The entire face must be in the photo.

3. Relative brightness $\mathrm{Y}>30$, according to the formula: $\mathrm{Y}=0.2126 * \mathrm{R}+0.7152 * \mathrm{G}+0.0722 * \mathrm{~B}$.

Where: R, G, B are the color values of the image taken in RGB space, respectively.

4. Open eyes with a straight face with a deviation between the cheeks of no more than $50 \%$.

We used a deep learning algorithm to detect 5 areas of the face (Eyes, eyebrows, nose, mouth) based on 68 points (17 points for face detection, 5 points for eyebrows, 6 points) identify one eye, 9 points identify nose, 20 points identify mouth). This result is used in the face detection process. The following image describes this process in detail. 
Detecting the area of interest In this step, the face image will be taken out of the background and focused only on the part with the face, the identification of 68 points will become more accurate. 68 points define specific parts of the face. Based on them, we was able to find 5 regions of interest (ROI) on the face such as forehead, nose, left and right cheeks, chin. Fixed ROI is important to provide an assessment of the size of the acne (number and size). Each ROI is dependent on the person, the distance from the camera to the face, the angle of the camera and the face in turn.

Histogram equalization Threshold equalization is a very important step because it will increase the contrast of the image. Since then, detecting acne in the following stages becomes easier.

Skin color segmentation The next step in the method is the acne detection process. In this system, the data analysis method used for acne detection is skin color segmentation. Acne is classified into 6 types, but according to the topic limit, the group will focus on detecting 4 basic types of acne (Hiền, 2020) include:

- Whiteheads (Non-inflammatory acne) are small pimples (1-2 mm).

- Blackheads (Non-inflammatory acne) are similar to whiteheads in that they are small (1-2mm) but open on the surface of the skin with dark areas appearing as black and brown.

- Papules (Inflammatory acne) is a type of acne that appears on the skin (1-4mm), with a hard pink bump color.

- Pustules (Inflammatory acne) is a type of acne that completely emerges as red inflammatory papules $(1-4 \mathrm{~mm})$, distinguished by a red center with a yellow or white cap in the center with a circle of lesions that are inflammatory.

To ensure less omission in acne detection, several color channels have been combined in the skin pixel segmentation. More specifically, the $\mathrm{a}^{*}$ channel is used to represent brightness and it can strengthen the red enhancement. The S channel in the HSV color channel is suitable for distinguishing between normal skin and whiteheads and blackheads.

Threshold Extraction Each threshold detects only a fraction of the total number of acnes on the ROI. This is because normal acne has many types and each of these types has its own color characteristics. In particular, the color values are different even with the same type of acne. So we suggested using a threshold list.

Queue processing A queue for each ROI generated. Each ROI is enqueued in this queue with the first threshold in the threshold list. ROI is converted from it to some areas that are highly likely to be acne. Then the ROI will be dequeue from the queue and these regions will be enqueued to the queue. These areas continue to be converted into smaller areas and are more likely to be acne. If any regions cannot continue to be converted, they are kept and re-queueed. Until we no longer have the threshold in the original threshold list, the process will be stopped.Noise removal processing

The output bowl area is usually detected in the system which may have noise or overlap with each other. We has implemented the Non Maximum Supression Algorithm with 2 noise type conditions: 1. If any detected area is larger than the maximum size of the acne or less than the minimum size of the acne. 2. If 2 or more areas overlap and the overlapping area covers more than $30 \%$, the overlapping but smaller areas will be eliminated. 


\subsection{Abnormal Points Classification}

\begin{tabular}{|c|c|}
\hline Layer & Type \\
\hline \multicolumn{2}{|c|}{ Input (RGB Image 30x30x3) } \\
\hline Block1Conv1 & Conv2D-32 \\
\hline Block1Conv2 & Conv2D-64 \\
\hline Activation Function & \\
\hline LeakyReLU & \\
\hline Block1Pool & Maxpooling2D \\
\hline Dropout1 & Dropout \\
\hline Block2Conv1 & Conv2D-128 \\
\hline Activation Function & \\
\hline LeakyReLU & \\
\hline Block2Pool & Maxpooling2D \\
\hline Block3Conv1 & Conv2D-128 \\
\hline Dense1 & Dense-64 \\
\hline Activation Function & \\
\hline LeakyReLU & \\
\hline Block3Pool & Maxpooling2D \\
\hline Flatten1 & Flatten-576 \\
\hline Dense2 & Dense-64 \\
\hline Dropout2 & Dropout \\
\hline Dense3 & Dense-6 (Softmax) \\
\hline
\end{tabular}

Table 1: Proposed CNN Architecture

Dataset ACNE04 dataset by Xiaoping Wu et al. (Wu, 2019) was proposed in 2019. The dataset is a collection of photographs of dermatological patients taken on one side of the face. There are 4 levels depending on the acne condition classified by the authors from more than 1000 photos, including level 0 (mild), level 1 (moderate), level 2 (severe), level 3 (very severe). However, the requirements for the CNN model are the types of acne, so we needs to conduct manual labeling on the image of one side of the face. There are 6 types of labels of different sizes and these 6 are equivalent to 6 types of photos that need to be classified as: whiteheads (490 photos), blackheads (490 photos), red inflammatory papules (490 photos), pustules ( 252 photos), normal skin (490 photos), non-skin photos (490 photos). From the above 6 labels, we built a binary classifier to classify acne or non-acne. In order not to lose generality, we grouped four types of acne into one class and the remaining types into one class. So the number of photos will include: photos with acne (1226 photos) and photos without acne (1226 photos).

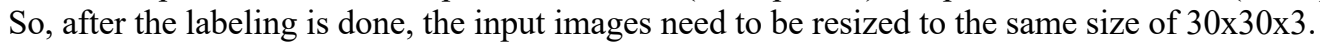

However, from the above number, we can easily realize that the big defect of the model lies in the fact that the training data is not large enough. So before putting it into the CNN model, we also needs to use data augmentation to avoid overfitting like the data enhancement techniques used: random rotation, displacement, division. scale, flip horizontal.

Proposed CNN Model The input to the model is 30x30 images that have been resized from the images after being obtained from the acne detection system. The model uses the LeakyRELU function on the entire system in order to avoid data loss. In the final layer of the model, it will be according to 
the binary classifier (for acne or non-acne) or 6 layers (whiteheads, blackheads, red inflammatory papules, pustules, normal skin, not skin) but Dense-6 or Dense- 2 .

The training model uses parameters such as: input size: 30x30x3, activation function: LeakyReLU, batchsize: 64, epoch: 40, using the optimal function Adam, using the loss function in the training process. is Multi-class Cross Entropy. The order of use of the 2 classifiers has a clear order. First, the outliers will go through the 6-class classifier first. Points that are predicted to be acne, will go through a 2-class classifier to ensure exactly that the point is acne.

\subsection{Level of Acne Evaluation Method}

The evaluation method used by we was improved from the Global Acne Grading (GAGS) method and the Hayashi acne counting method (mentioned in Chapter II.1.2) to fit the output of the classification problem. The type is mGAGS (Modified GAGS). This method applies weights to different types of acne as follows: whiteheads - 1 , blackheads - 1 , red inflammatory papules -2 , pustules - 3. From there, we proposed the formula to be converted as follows:

$$
\begin{aligned}
& \text { Where: } 0<=\mathrm{S}<=5 \text { : mild, } \\
& 6<=\mathrm{S}<=20 \text { : moderate, } \\
& 21<=\mathrm{S}<=50 \text { : severe, } \\
& \mathrm{S}>50 \text { : very severe with } \mathrm{S} \text { being the score, } \\
& \text { WH is number of whiteheads, } \\
& \text { BH is number of blackheads, } \\
& \text { PA is number of papules, } \\
& \text { PU is number of pustules. }
\end{aligned}
$$

\subsection{Recommendation System}

Dataset The data used for product and route recommendations was obtained by we from the cosmetics e-commerce site Hasaki. Using the Python powered Selenium library, we managed to get close to 1000 products of all kinds.

Automatic product suggestion Automatic product suggestion will be implemented by hybrid system. This system will use after the user takes a picture and has received the results returned from the previous realizers. The group's suggestion system consists of 2 main steps: step 1: Filtering by knowledge domain, step 2: Filtering with hybrid.

Step 1: Filter by knowledge domain

The system will search for products with components that match the current status of the user. If the user has non-inflammatory acne, the system will filter products with the following ingredients: Salicylic acid, Retinoids, Azelaic acid, Benzoyl peroxide. And if the user has inflammatory acne, the products have the following ingredients: Retinoids, Azelaic acid, Benzoyl peroxide.

From there, the system will have a list of how products and will be calculated and classified in the next steps.

Step 2: Filter with hybrid

The products will be passed through the system of suggested hybrid, weighted hybrid and suggested hybrid. The product recommendation is based on the method of hybrid recommender system including: weighted hybrid, augmented hybrid. Weighted hybrid means that the group puts weight on some field of the product in order to create a new attribute to suggest on that attribute. The weights of the product fields are weighted according to their importance in the recommender system as described above. The weights of the fields that sum to 1 to ensure fairness in the calculation are Score. So, the Score field is calculated by the formula: 
Score $=$ Good_ingredient $* 0.15+$ Price $*(-0.15)+$ Rating $* 0.15+$ Comment $* 0.1+$ Good_Comment $* 0.15+$ Favorite $* 0.15+$ Recommend $* 0.2+$ Effective $* 0.2$

From there, the product will be filtered and sorted according to the enhancement field from high to low. In the last step, the system has obtained a list of products that match the user's status, as well as based on similar users and products with high enhancement fields at the current time. We will send a list of products to the user.

Proactive Product Suggestions Manual product suggestions fall into two categories. Type 1: when the user has not defined the product he wants. Type 2: when you have determined the product you want.

When the user has not identified the product he wants

When the user has not identified the product but only types a few characters, the system will use Full-text Search to suggest products by keyword, which is the name of the product quickly. as quickly as possible.

When you have determined the product you want.

Once you have determined the product you want, the system will suggest similar products that the user is looking for by using the similarity table between products to return the 10 most similar products. with the product you are looking for.

Support to suggest a treatment route Based on the knowledge we learned, the research team proposed a general 5-step skin care process in the morning and general 5 steps at night.

Morning - step 1: Cleanser, step 2: Toner, step 3: Serum - Essence, step 4: Cream - nourishing oil, step 5: Sunscreen product.

Evening - step 1: Remove makeup, step 2: Cleanser, step 3: Exfoliate, step 9: Toner, step 10: Reduce acne - fade dark spots.

\section{Result And Discussion}

\begin{tabular}{llcccc}
\hline No. & Type of acne & Acne Total & Acne TP & Acne FN & Acne Sensitivity \\
\hline 1 & Whiteheads & 444 & 396 & 48 & 89.19 \\
2 & Blackheads & 588 & 518 & 70 & 88.10 \\
3 & Inflammatory acne & 345 & 317 & 28 & 91.88 \\
Total & & 1377 & 1231 & 146 & 89.4 \\
\hline
\end{tabular}

Table 2: Result of level of acne evaluation

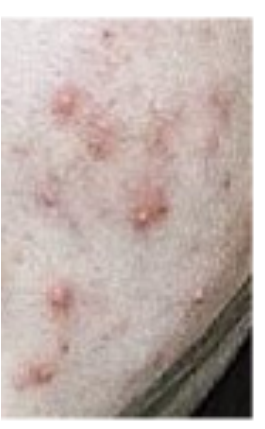

(a)

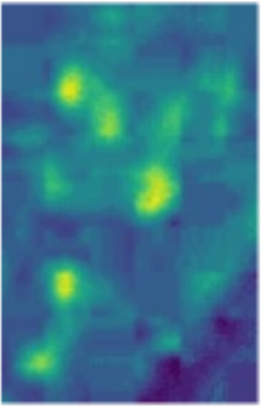

(b)

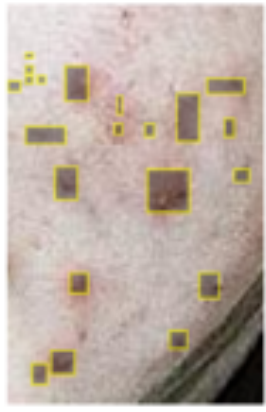

(c)

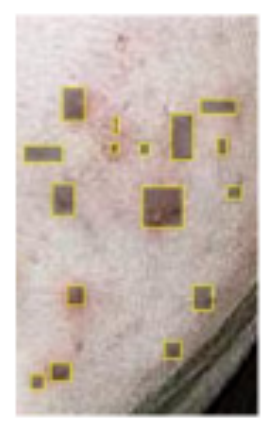

(d)

Figure 1. Standard acne detection procedure 
By using the Recall evaluation index to evaluate the anomaly detection pot, the group obtained the following results. The acne in picture (a) is mostly inflammatory and they appear to be large and numerous. In figure (b), this image has been converted from the original RGB image to one that uses channel $\mathrm{a}^{*}$ in the CIELa*b* color space. This transformation makes the inflammatory acne appear brighter in the picture. Threshold processing (c) makes color apparent in the detection of inflammatory acne. However, the problem here is that extremely small squares are also considered as pimples but they are not actually pimples. These regions are removed after noise filtering (d) is applied.

\begin{tabular}{llllllll}
\hline Metric & $\begin{array}{c}\text { Precision } \\
\uparrow\end{array}$ & $\begin{array}{c}\text { Recall } \\
\uparrow\end{array}$ & $\begin{array}{c}\text { F1- } \\
\text { Score } \\
\uparrow\end{array}$ & $\begin{array}{c}\text { Trainning } \\
\text { accuracy } \uparrow\end{array}$ & $\begin{array}{c}\text { Tranining } \\
\text { loss } \downarrow\end{array}$ & $\begin{array}{c}\text { Testing } \\
\text { accuracy } \\
\uparrow\end{array}$ & $\begin{array}{c}\text { Testing } \\
\text { loss } \\
\downarrow\end{array}$ \\
\hline $\begin{array}{l}\text { Model } \\
\text { Shen }\end{array}$ & 92.0 & 91.5 & 92.0 & 92.05 & 24.37 & 92.17 & 24.86 \\
$\begin{array}{l}\text { Model } \\
\text { Cheeoh }\end{array}$ & 86.0 & 86.0 & 85.5 & 89.23 & 33.1 & 86.9 & 33.28 \\
$\begin{array}{l}\text { VGG16 } \\
\text { Fine-tuning }\end{array}$ & 92.0 & 92.0 & 92.0 & 92.23 & 21.14 & 92.19 & 21.05 \\
$\begin{array}{l}\text { VGG16 } \\
\begin{array}{l}\text { SVM } \\
\text { Our 2- } \\
\text { classes } \\
\text { model }\end{array}\end{array}$ & 91.0 & 91.0 & 91.0 & 91.22 & $\mathrm{NaN}$ & 91.26 & $\mathrm{NaN}$ \\
\hline
\end{tabular}

Table 3: Result of 2-classes classification

\begin{tabular}{lllllllc}
\hline Metric & $\begin{array}{c}\text { Precision } \\
\uparrow\end{array}$ & $\begin{array}{c}\text { Recall } \\
\uparrow\end{array}$ & $\begin{array}{c}\text { F1- } \\
\text { Score } \\
\uparrow\end{array}$ & $\begin{array}{c}\text { Trainning } \\
\text { accuracy } \uparrow\end{array}$ & $\begin{array}{c}\text { Tranining } \\
\text { loss } \downarrow\end{array}$ & $\begin{array}{c}\text { Testing } \\
\text { accuracy } \\
\uparrow\end{array}$ & $\begin{array}{c}\text { Testing } \\
\text { loss } \\
\downarrow\end{array}$ \\
\hline $\begin{array}{l}\text { Model } \\
\text { Shen }\end{array}$ & 79.17 & 77.33 & 77.83 & 79.4 & 57.8 & 79.26 & 57.34 \\
$\begin{array}{l}\text { Model } \\
\text { Cheeoh }\end{array}$ & 76.5 & 71.83 & 73.0 & 74.39 & 71.08 & 74.13 & 71.05 \\
$\begin{array}{l}\text { VGG16 } \\
\text { Fine-tuning }\end{array}$ & 77.67 & 75.0 & 75.67 & 78.31 & 61.21 & 78.19 & 61.26 \\
$\begin{array}{l}\text { VGG16 } \\
\text { SVM }\end{array}$ & 78.83 & 77.67 & 78.0 & 80.37 & NaN & 80.33 & NaN \\
$\begin{array}{l}\text { Our 6- } \\
\text { classes } \\
\text { model }\end{array}$ & $\mathbf{8 4 . 1 7}$ & $\mathbf{8 1 . 5}$ & $\mathbf{8 2 . 0}$ & $\mathbf{8 4 . 1 8}$ & $\mathbf{4 7 . 2 9}$ & $\mathbf{8 4 . 1}$ & $\mathbf{4 7 . 3 9}$ \\
\hline
\end{tabular}

Table 4: Result of 6-classes classification 


\begin{tabular}{llll}
\hline Metric & $\begin{array}{c}\text { Accuracy } \\
\text { of level 0 } \\
\uparrow\end{array}$ & $\begin{array}{c}\text { Accuracy } \\
\text { of level 1 } \\
\uparrow\end{array}$ & $\begin{array}{c}\text { Average } \\
\text { accuracy } \\
\uparrow\end{array}$ \\
\hline Student 1 & 43.75 & 23.78 & 31.88 \\
Student 2 & 51.25 & 39.84 & 45.63 \\
Student 3 & 66.25 & 30.72 & 48.12 \\
Student 4 & 77.5 & 41.68 & 56.25 \\
Student 5 & 42.5 & 18.56 & 28.75 \\
Student 6 & 65.0 & 25.38 & 42.5 \\
Hayashi & 91.25 & 73.46 & 78.13 \\
GAGS & 97.5 & 58.59 & 74.38 \\
Our method & $\mathbf{9 1 . 2 5}$ & $\mathbf{7 8 . 6 3}$ & $\mathbf{8 1 . 2 5}$ \\
\hline
\end{tabular}

Table 5: Result of level of acne evaluation method

\begin{tabular}{|c|c|c|c|c|c|c|c|c|}
\hline & $\begin{array}{c}\text { Femal } \\
\text { e }\end{array}$ & $\begin{array}{c}\text { Femal } \\
\text { e }\end{array}$ & $\begin{array}{c}\text { Femal } \\
\text { e }\end{array}$ & Female & Male & Male & Male & Male \\
\hline & Norma & Oil & Mixed & Sensitiv & Norma & Oil & Mixe & Sensitiv \\
\hline & 1 Skin & Skin & Skin & e Skin & 1 Skin & Skin & $\begin{array}{l}\mathrm{d} \\
\text { Skin }\end{array}$ & e Skin \\
\hline Cleaner & 0.7 & 0.73 & 0.73 & 0.75 & 0.8 & 0.78 & 0.8 & 0.9 \\
\hline Toner & 0.75 & 0.83 & 0.7 & 0.8 & 0.78 & 0.7 & 0.78 & 0.7 \\
\hline Serum & 0.73 & 0.7 & 0.8 & 0.7 & 0.75 & 0.73 & 0.7 & 0.83 \\
\hline $\begin{array}{l}\text { Cream - } \\
\text { nourishing oil }\end{array}$ & 0.7 & 0.7 & 0.75 & 0.73 & 0.83 & 0.75 & 0.78 & 0.73 \\
\hline $\begin{array}{l}\text { Remove } \\
\text { makeup }\end{array}$ & 0.8 & 0.78 & 0.8 & 0.83 & 0.8 & 0.85 & 0.83 & 0.85 \\
\hline Exfoliate & 0.7 & 0.8 & 0.73 & 0.78 & 0.8 & 0.83 & 0.85 & 0.85 \\
\hline $\begin{array}{l}\text { Reduce acne - } \\
\text { fade dark }\end{array}$ & 0.81 & 0.75 & 0.75 & 0.81 & 0.88 & 0.81 & 0.88 & 0.88 \\
\hline
\end{tabular}

With the 2-class classifier, we can realize the insignificant difference between the models, however, with the 6-class classifier, the model proposed by the group has the highest accuracy value and the loss Also less than the rest of the models.

The results of the group's acne assessment achieved an accuracy of $81.25 \%$, which was higher than the average accuracy of the medical students, also known as the common subjects. In addition, the group's evaluation method, although at level 0 is still not suitable, but at level 1 , the accuracy is quite high and the average accuracy is higher than that of Hayashi's evaluation method and GAGS method. From the above two things, it is proved that on this data set, the proposed group method to evaluate the degree of acne is appropriate.

We will rate based on the products recommended by the system that have ingredients recommended by the doctor. Specifically, there will be 4 acne conditions being encountered (mild, moderate, severe, very severe), 4 skin types of the user (normal skin, oily skin, sensitive skin, combination skin) and 2 gender types. gender (male, female). The suggested method achieved high results, based on the method proposed by the group 


\section{Conclusion}

The research project "Application in the assessment of acne status and supporting route suggestion" is a complete solution and can be put into practice. The solution provides 4 processors in the same solution to fully meet the original problem posed by we. The accuracy in each set is at a stable level and the first results in practical application have been obtained with nearly 1000 uses. However, the work still has some limitations such as the fact that the detector is still light dependent and the response time needs to be faster. At the same time, the product suggestion set needs to be personalized for the user.

\section{Conflicts of Interest}

The authors declare no conflicts of interest.

\section{Acknowledgment}

We acknowledge the support of time and facilities from Ho Chi Minh City University of Technology (HCMUT) - VNU-HCM for this study.

\section{References}

Cheeoh, J. (30 April 2019). Classification Model of Facial Acne Using Deep Learning. Journal of the Korea Institute of Infomation and Communication Engineering.

Gyeongeun Lee, D. o. (2020). A Content-based Skincare Product Recommendation System.

Hiền, T. N. (2020). Nghiên cứu độc tính và hiệu quả của ACNECA trong việc điều trị bệnh trứng cá thông thường thể vừa.

Mohammad Amini, F. V. (22 February 2018). Automated facial acne assessment from smartphone images. Proc. SPIE 10497, Imaging,Manipulation, and Analysis of Biomolecules, Cells, and Tissues XVI.

Nobukazu Hayashi, H. A. (2008). Establishment of grading criteria for acne severity. The Journal of Dermatology.

R. Ramli, A. S. (Feb. 2012). Acne analysis, grading and computational assessment methods: an overview. Skin Res. Technol., vol. 18.

Sáu, N. H. (2010). Cập nhật điều trị bệnh trứng cá. Tạp chí thông tin Y-Dược, 7, 2-6.

Wu, X. (2019). Joint Acne Image Grading and Counting via Label Distribution Learning.

Xiaolei Shen, J. Z. (11 April 2018). An Automatic Diagnosis Method of Facial Acne Vulgaris Based on Convolutional Neural Network. 